\title{
Automatic Analysis of Pediatric Renal Ultrasound Using Shape, Anatomical and Image Acquisition Priors
}

\author{
Carlos S. Mendoza ${ }^{1,2}$, Xin Kang ${ }^{1}$, Nabile Safdar ${ }^{1}$, \\ Emmarie Myers ${ }^{1}$, Aaron D. Martin ${ }^{1,3}$, Enrico Grisan ${ }^{4}$, \\ Craig A. Peters ${ }^{1,3}$, and Marius George Linguraru ${ }^{1}$ \\ 1 Sheikh Zayed Institute for Pediatric Surgical Innovation, Children's National \\ Medical Center, Washington DC, USA \\ carlos.sanchez.mendoza@gmail.com \\ 2 Signal Processing Department, University of Sevilla, Spain \\ 3 Division of Urology, Children's National Medical Center, Washington DC, USA \\ 4 Department of Information Engineering, University of Padova, Italy
}

\begin{abstract}
In this paper we present a segmentation method for ultrasound (US) images of the pediatric kidney, a difficult and barely studied problem. Our method segments the kidney on 2D sagittal US images and relies on minimal user intervention and a combination of improvements made to the Active Shape Model (ASM) framework. Our contributions include particle swarm initialization and profile training with rotation correction. We also introduce our methodology for segmentation of the kidney's collecting system (CS), based on graph-cuts (GC) with intensity and positional priors. Our intensity model corrects for intensity bias by comparison with other biased versions of the most similar kidneys in the training set. We prove significant improvements $(p<0.001)$ with respect to classic ASM and GC for kidney and CS segmentation, respectively. We use our semi-automatic method to compute the hydronephrosis index (HI) with an average error of $2.67 \pm 5.22$ percentage points similar to the error of manual HI between different operators of $2.31 \pm 4.54$ percentage points.
\end{abstract}

\section{Introduction}

Kidney segmentation in US images is a topic of limited presence in the image processing literature [1,2]. Several limitations of US images make segmentation a particularly daunting task: poor signal-to-noise ratio, signal drop-out, missing boundaries, misplaced boundaries and reconstruction errors [3]. US segmentation methods have been previously classified according to the prior knowledge employed to improve the accuracy of results. These constraints include image-derived priors (intensity, intensity derivatives, local phase, texture), and application-derived priors (shape and motion) [3].

Two-dimensional ultrasound (US) is the widely preferred image modality for in vivo assessment of renal conditions, particularly for pediatric applications, mainly for its safety and cost-effectiveness, both prenatally and postnatally. 
For the diagnosis of hydronephrosis (dilatation of the renal pelvis and calyces, often caused by obstruction of the free flow of urine from the kidney [4]) in young children, Shapiro et al. [5] have proposed a hydronephrosis index (HI), a quantitative measure in which HI (percentage $)=100 \times($ renal area - renal pelvis $/$ calyces area)/(renal area). Computation of HI requires manual delineations of the kidney and the pelvis/calyces on a single 2D sagittal US image in which the kidney achieves its maximal longitudinal dimensions. It could be discussed whether using a single slice allows for robust shape model creation and good estimation of HI. We show in Fig. 3 in Section 3 some examples of longitudinal kidney sections and in Fig. 1 the good behaviour of the obtained shape model can be appreciated. Although the estimation of HI would certainly be improved by 3D quantification, it is not our purpose to go beyond the accuracy of the current standard clinical procedure, which is performed on properly chosen $2 \mathrm{D}$ slices due to higher availability of $2 \mathrm{D}$ acquisition equipment [5].

The purpose of the work presented herein is to increase the availability and reduce the variability of $\mathrm{HI}$ computation by automating the segmentation of the renal parenchyma and the renal collecting system (pelvis and calyces). This task presents several challenges. For the segmentation of renal structures there is limited availability of robust priors, especially from noisy 2D US images acquired at variable angles that give variable renal shapes. Furthermore, in US the interior of the kidney exhibits heterogeneous structures of different shapes, sizes and intensities, and many of the boundaries are lost due to the density similarity to surrounding structures and shadowing [1.

To the best of our knowledge, the automated segmentation of the renal collecting system (CS) has not been tackled by previous work. CS appears as a hypoechoic (dark) region inside the kidney, and expands into the parenchyma from the ureteropelvic junction (UPJ). Difficulties for segmentation of CS in sagittal US sections arise due to intensity offset, signal drop-out (shadowing) and loss of the 3D connectivity of the CS region when studied on 2D views. Furthermore, in very young children the renal pyramids appear more hypoechoic than in other subjects, and can be confused with CS even by the experienced eye.

In the following Sections we present our methodology for segmentation of renal structures relevant to hydronephrosis. For segmenting the kidney we introduce a modification of the classic Active Shape Models (ASM) [6], with shape and radial intensity priors based on statistical models that incorporate the relative orientation with respect to the US probe. We also propose an automatic robust initialization strategy, important for ASM under large shape uncertainty, based on particle swarm optimization. For segmentation of CS we introduce a methodology based on graph-cuts (GC) [7] with intensity and positional priors. To correct for intensity bias we developed a correction strategy based on probability density function (PDF) cross-correlations with similar cases identified in the training set. As output of the GC algorithm we obtain a set of candidate regions that are ranked according to anatomical and clinical priors. 


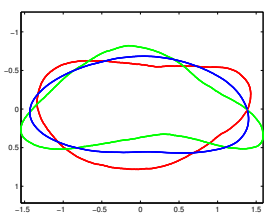

(a)

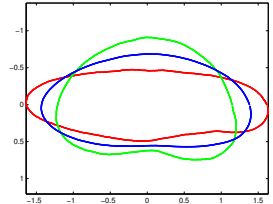

(b)

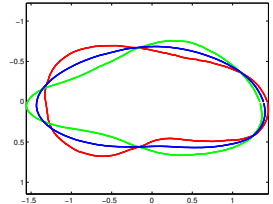

(c)

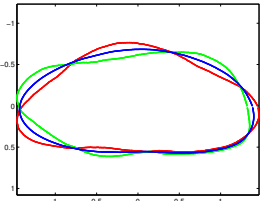

(d)

Fig. 1. Three principal modes of shape variations for the kidney shape model. (a)-(d) First to forth mode. Blue: mean shape. Green: mean $+3 \sigma$. Red: mean $-3 \sigma$.

Ultimately, the proposed technique allows the semi-automatic analysis of the kidney and its CS from difficult US image data with low error.

\section{Method}

\subsection{Kidney Segmentation: ASM with Image Acquisition Priors}

In the classic ASM methodology [6], shape is represented by a large number of landmarks distributed along the object's contour. ASM performs by adjusting the position of the landmarks to fit pre-trained radial intensity derivatives along rays traced normally to the contour's landmarks. The new set of landmarks is then filtered through the pre-trained shape model. This procedure is re-iterated until convergence or for a given number of iterations. The shape model does not incorporate any information on the pose, particularly relevant in US images, but is instead constructed from pose-corrected instances. Our pose normalization was performed by finding the centroid of the region enclosed by the contour, by correcting orientation using the main axis of inertia, and by scaling the contour according to the average distance from the computed centroid. Then landmarks were obtained by even arc-length sampling of the contour. Furthermore, in ASM the range of search on the radial profiles is usually small. Therefore, a good initial estimation of the pose (location, orientation, scale) of the object is necessary for good convergence. In our method, the user provides two mouse-clicks along the main longitudinal axis of the kidney so that the line connecting the two clicks provides an initial approximation of centroid location (midpoint), orientation and scale.

Particle Swarm Optimization (PSO) [8] is adopted to optimize the initializing shape for the ASM (the pose, and also the shape by allowing variations along the main modes of variation). PSO was chosen because it makes few or no assumptions about the problem being optimized and can search very large spaces of candidate solutions. In PSO the optimization is achieved by having a swarm of particles (candidate solutions) moving around in the search space. Each particle's position and velocity are initialized as uniformly distributed random vectors and each particle's position and velocity are updated according to the best solutions found in previous iterations. In our implementation the pose is obtained from the 


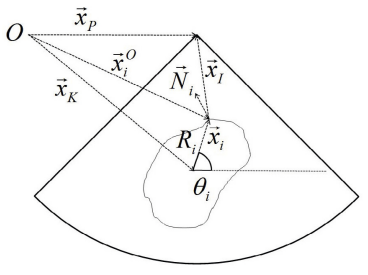

(a)

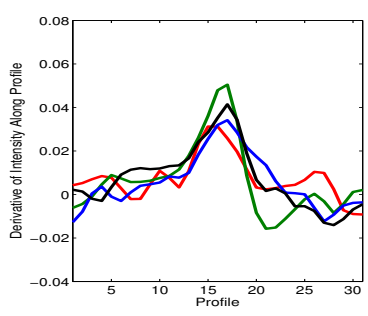

(b)

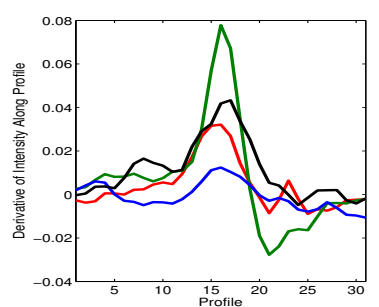

(c)

Fig. 2. (a) Definitions for computation of the incidence angle. Edge profiles without (b) and with (c) rotation correction averaged across all kidneys for landmarks spaced around the kidney contours $\left(\theta_{i}=(0\right.$ (red), $\pi / 2$ (green), $\pi$ (blue), $3 \pi / 2$ (black)).

two mouse-clicks as described above, allowing variations of \pm 20 pixels in location, \pm 10 degrees in orientation, and \pm 10 in scale. The result is a pose/shape-adjusted instance of the shape model that best fits the image features. All parameters in this and following algorithms were tuned heuristically by systematic trial and error, and further parameter space exploration was left for future work.

Both the initialization and ASM procedures rely on the sum of Mahalanobis distances between the radial intensity derivative profiles at landmark locations for the test image and training data. In our adaptation to US, in order to account for the fading of edges that are tangent to the US propagation direction, profile training must be addressed not by anatomical landmark correspondence, but instead in accordance to the angle of incidence at landmark $i$, located at $x_{i}$. According to the conventions in Fig. 2(a) referred to image origin $O$, if the position of the probe $\left(x_{P}\right)$ and the kidney centroid $\left(x_{K}\right)$ are considered approximately constant for all images, the incidence direction only depends on $x_{i}$. Then if we approximate the contour of the kidney by a circumference of radius $R$, both the normal $N_{i} \approx \frac{x_{i}}{\left|x_{i}\right|}$ and $x_{i} \approx\left(R \cos \theta_{i}, R \sin \theta_{i}\right)$ (and therefore $x_{I}$ ) are only a function of the angular coordinate $\alpha_{i} \approx f\left(\theta_{i}\right) \approx f\left(i-i_{0}\right)$, where landmark $i_{0}$ has angular coordinate $\theta_{0}$. Then, the right incidence-angle correspondence can be obtained if the set of landmarks is circulated around the contour according to the orientation $\theta_{0}$ of every shape instance. As seen in Fig. 1(b-c), the kidney radial profiles become more distinct when rotation correction is applied, since the averaging over landmarks with different incidence angles is avoided.

\subsection{CS Segmentation: Intensity Correction and Anatomical Priors}

In renal US the structures of the CS appear as hypoechoic structures proximal to the UPJ (Fig. 3). To incorporate priors of intensity and position an appropriate framework is GC segmentation [7]. GC techniques operate on a graph analogue of an image and exhibit good performance and guaranteed convergence of energies of the following form: $E(f)=\Sigma_{p \in P}\left(D_{p}\left(f_{p}\right)\right)+\lambda \Sigma_{(p, q) \in N}\left(V_{p, q}\left(f_{p}, f_{q}\right)\right)$, where $D_{p}(\cdot)$ is the pixel-wise regional term and $V_{p, q}(\cdot, \cdot)$ is the boundary term for 
measuring the interaction potential over neighborhood $N$, for a labeling scheme $f$. Often, the regional term depends on the Bayesian likelihood of a certain pixel feature according to a pre-trained model. We propose nonparametric estimation of the likelihoods for intensity and also position via kernel density estimation (KDE) from training images.

Intensity Model. The main characteristic of the CS in US images is its hypoechoic appearance. However, US images can present significant intensity bias due to user-controlled depth gain settings and beam attenuation within the body. Other authors proposed intensity correction for US images by estimating the bias field according to complex models [9. We make a less restrictive assumption: that the intensity distribution of the kidney suffers from a constant bias that can be determined by comparison with biased versions training kidneys. The advantage is that no assumptions are made on specific bias field models.

For each of the test images we estimate the PDF of the pixels inside the kidney according to the automatic segmentations performed as in Section 2.1 The intensity distributions of the training images for the pixels inside the kidney, parenchyma and CS are obtained according to manual segmentations from our ground truth. If $f_{i}$ is the PDF of kidney intensities in training image $i$ we define the bias $b_{i}$ as

$$
b_{i}=\underset{|n| \leq Q}{\operatorname{argmax}}\left(f_{i} \star g\right)[n],
$$

where $g$ is the kidney PDF in the test image, and $\star$ denotes cross-correlation. Notice that the cross-correlation is maximized over bounded values of $n$. If $\left\{C_{j}^{g}\right\}_{j=1 \ldots K}$ are the $K$ (we used $K=10$ ) training images with larger $\left(f_{i} \star g\right)\left[b_{i}\right]$ among the $T$ training images $\left\{C_{i}\right\}_{i=1 \ldots T}$, then our intensity models $\hat{f}_{g}^{P / C S}$ for segmenting image $g$ into parenchyma or collecting system $(P / C S)$ are obtained as

$$
\hat{f}_{g}^{P / C S}=\operatorname{KDE}\left(\left\{\left\{I_{k, C_{j}^{g}}^{P / C S}+b_{C_{j}^{g}}\right\}_{k=1 \ldots M_{C_{j}^{g}}^{P / C S}}\right\}_{j=1 \ldots K}\right)
$$

where $I_{k, C_{j}^{g}}^{P / C S}$ is the $k$-th intensity sample over $P / C S$ for training image $C_{j}^{g}$, from the set of all $M_{C_{j}^{g}}^{P / C S}$ samples. Intuitively, we find a subset of the most similar training kidneys and for which bias this highest similarity occurs for each one of them. Then the intensity model is obtained from kernel density estimation over the bias-corrected training kidneys in the obtained subset.

Position Model. Another available prior for CS segmentation is its anatomical location with respect to the UPJ. The location of UPJ for each kidney is obtained by exploiting landmark correspondence in the ASM. To build the positional model from kidneys of different sizes we normalize the distances from each pixel to the UPJ by the distance from the kidney centroid to UPJ, in each of the $T$ training segmentations (ground truth). The normalized positional models 
$\hat{p}^{P / C S}$ for $P / C S$ segmentation are estimated from the set of all normalized pixel locations $J_{k, i}^{P / C S}$ of each training image $i$ as

$$
\hat{p}^{P / C S}=\operatorname{KDE}\left(\left\{\left\{J_{k, i}^{P / C S}\right\}_{k=1 \ldots M_{i}^{P / C S}}\right\}_{i=1 \ldots T}\right) .
$$

Graph Energy. The trained models for intensity and position can be incorporated into the region term of the GC energy:

$$
D_{p}(P / C S)=\hat{f}_{g}^{P / C S}\left(I_{p}\right)+\alpha \cdot \hat{p}^{P / C S}\left(J_{p}\right) .
$$

The boundary term depends on the difference of likelihood for intensities across the n-link to being CS:

$$
V_{p, q}\left(f_{p}, f_{q}\right)= \begin{cases}\beta \cdot e^{\gamma\left|\hat{f}_{g}^{C S}\left(I_{p}\right)-\hat{f}_{g}^{C S}\left(I_{q}\right)\right|} & \text { if } f_{p} \neq f_{q} \\ 0 & \text { otherwise }\end{cases}
$$

$\alpha, \beta$ and $\gamma$ are tuning constants (we used $\alpha=0.5, \beta=5$ and $\gamma=100$ ) that weight the contributions of the different terms to the GC energy.

Candidate Selection. The output of the GC segmentation can consist of several regions. However, CS is a single connected structure inside the kidney, so we post-process the results by connected-component analysis. For each of the $L$ output connected components $\left\{C C_{l}\right\}_{l=1 \ldots L}$ we compute a score $S\left(C C_{l}\right)$ as follows:

$$
\begin{aligned}
S\left(C C_{l}\right) & =\overline{\left(1-\left|\frac{M^{C C_{l}}}{M^{K}}\right|\right)}+\delta \overline{\left(\cdot \frac{\operatorname{Mean}\left(\left\{I_{k}^{C C_{l}}\right\}_{\left.k=1 \ldots M^{C C_{l}}\right)}\right.}{\operatorname{Mean}\left(\left\{I_{k}^{K}\right\}_{k=1 \ldots M^{K}}\right)}\right)}+ \\
& +\epsilon \cdot \overline{\operatorname{Mean}\left(\left\{J_{k}^{C C_{l}}\right\}_{\left.k=1 \ldots M^{C C_{l}}\right)}\right.},
\end{aligned}
$$

where $M^{A}$ is the number of pixels in region $A$, and $K=P \cup C S$ (i.e. the kidney). The bar indicates the normalization of the mean and standard deviation of the terms to the $[0,1]$ range over all connected components $\left\{C C_{l}\right\}_{l=1 \ldots L}$. We used $\delta=0.9$ and $\epsilon=0.3$. Intuitively, the score $S$ is a measure of likelihood for a region to be selected as final single connected segmentation among the regions obtained from GC. It integrates priors on region size (should be large), region relative hypoechoic quality (should be high) and location relative to UPJ (should be close).

\section{Results}

We collected 34 US image studies of hydronephrotic kidneys, from one Philips IU22 and three General Electrics Logiq E9 units. Two trained operators (GT1 and GT2) manually delineated the kidneys and their CS, under the supervision 


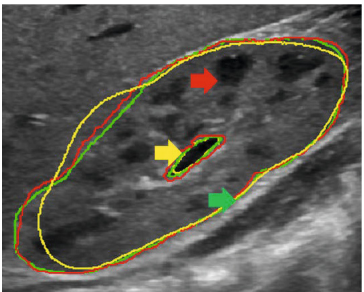

(a)

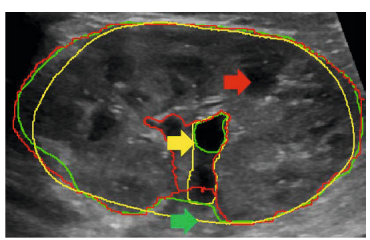

(d)

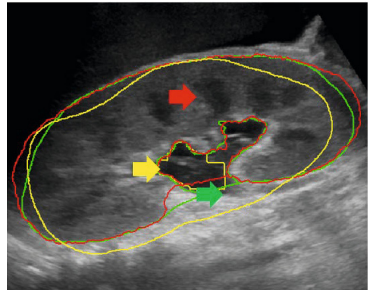

(b)

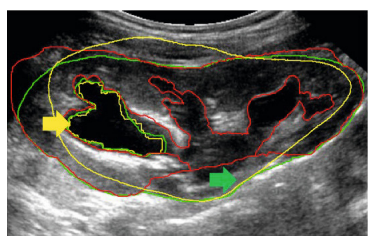

(e)

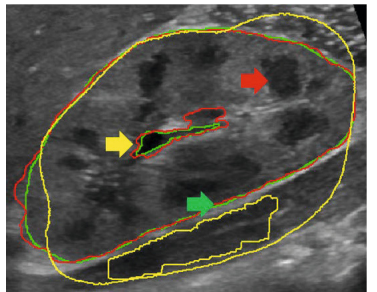

(c)

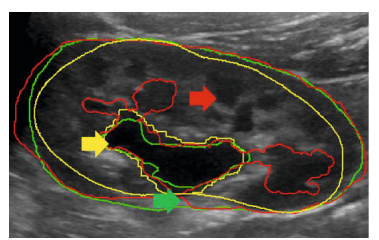

(f)

Fig. 3. Results for (a) best, (b) median and (c) worst case. (d-f) Some more results. Green: GT1. Red: GT2. Yellow: Result. Green arrow: UPJ. Red arrow: Renal pyramid. Yellow arrow: CS.

Table 1. DICE, RAD, and MPD (pixels) with respect to GT1. IO K: Kidney delineation by GT2. cKS: Kidney segmentation with classic ASM. KS: Kidney segmentation with proposed method. IO CS: CS delineations by GT2. cCS: CS segmentation with classic GC. CS: CS segmentation with proposed method.

\begin{tabular}{|c||c|c|c|c|c|c|}
\hline Measure & IO K & cKS & KS & IO CS & cCS & CS \\
\hline \hline Dice & $0.95 \pm 0.03$ & $0.83 \pm 0.04$ & $0.87 \pm 0.05$ & $0.76 \pm 0.18$ & $0.32 \pm 0.22$ & $0.62 \pm 0.32$ \\
\hline RAD & $0.04 \pm 0.04$ & $0.16 \pm 0.11$ & $0.13 \pm 0.11$ & $0.79 \pm 1.48$ & $1.42 \pm 1.54$ & $0.85 \pm 1.21$ \\
\hline MPD & $6.47 \pm 4.14$ & $21.2 \pm 6.36$ & $17.3 \pm 7.50$ & $11.7 \pm 14.1$ & $28.4 \pm 41.4$ & $25.3 \pm 35.3$ \\
\hline
\end{tabular}

of a radiologist and a urologist, on a single sagittal 2D US image. All images and GT1 delineations were used for training and testing in a leave-one-out configuration. The average time for each test was $45 \pm 39 \mathrm{~s}$. (ASM) and $4 \pm 2 \mathrm{~s}$. (GC).

We compared results for kidney and CS segmentation from our methods (M) with GT1, and also compared GT2 with GT1. We obtained Dice's coefficients (DICE), relative area differences (RAD), and mean perimeter distances (MPD). Classic ASM (mean shape initialization, no rotation correction) and classic GC (no bias correction, positional prior or candidate selection) implementations were also included in the comparison. Results are shown in Table 1. Significant improvements $(p<0.001)$ were noted using our method over a classic ASM (for kidney segmentation) and classic GC (for CS segmentation). We computed HI for all test cases as in [5] using GT1, GT2 and M. The average error for M vs. GT1 was $2.67 \pm 5.22$ percentage points, and $2.31 \pm 4.54$ for GT2 vs. GT1. HIs from M and GT1 are significantly correlated $(r=0.99, p<0.001)$. Fig. 3 shows segmentation results. 


\section{Conclusion}

We have presented a segmentation framework for renal US. Our kidney segmentation method is based on an improvement of ASM via rotation correction and PSO initialization. Our framework also includes a GC segmentation algorithm for structures of the renal collecting system (pelvis and calyces). The GC energy depends on intensity and positional priors estimated from training images via KDE. Intensity bias is corrected by optimizing cross-correlations of the test image PDF with the most similar kidneys in the training set. Results show that our approach performs well in difficult images, and that the HI can be obtained with error similar to the disagreement between manual operators, suggesting the potential for computer-aided diagnosis of (pediatric) renal conditions, related to the size and geometry of the organ and its substructures, from US imaging data.

Acknowledgment. This project was supported by a philanthropic gift from the Government of Abu Dhabi to Children's National Medical Center. Its contents are solely the responsibility of the authors and do not necessarily represent the official views of the donor.

\section{References}

1. Martín-Fernández, M., Alberola-Lopez, C.: An approach for contour detection of human kidneys from ultrasound images using Markov random fields and active contours. Med. Image. Anal. 9(1), 1-23 (2005)

2. Xie, J., Jiang, Y., Tsui, H.: Segmentation of kidney from ultrasound images based on texture and shape priors. IEEE Trans. Med. Imag. 24(1), 45-57 (2005)

3. Noble, J.: Ultrasound image segmentation and tissue characterization. Proc. Inst. Mech. Eng. H J. Eng. Med. 224(2), 307-316 (2010)

4. Peters, C., Chevalier, R.: Congenital urinary obstruction: Pathophysiology and clinical evaluation. In: Wein, A., Kavoussi, L., Novick, A., Partin, A., Peters, C. (eds.) Campbell-Walsh Textbook of Urology. Elsevier Inc., Philadelphia (2012)

5. Shapiro, S., Wahl, E., Silberstein, M., Steinhardt, G.: Hydronephrosis index: A new method to track patients with hydronephrosis quantitatively. Urology 72(3), 536$538(2008)$

6. Cootes, T.F., Edwards, G.J., Taylor, C.J.: Active appearance models. In: Burkhardt, H., Neumann, B. (eds.) ECCV 1998. LNCS, vol. 1407, pp. 484-498. Springer, Heidelberg (1998)

7. Boykov, Y., Veksler, O., Zabih, R.: Fast approximate energy minimization via graph cuts. IEEE Trans. Pattern Anal. Mach. Intell. 23(11), 1222-1239 (2001)

8. Clerc, M., Kennedy, J.: The particle swarm - explosion, stability, and convergence in a multidimensional complex space. IEEE Trans. Evol. Comput. 6(1), 58-73 (2002)

9. Xiao, G., Brady, M., Noble, J., Zhang, Y.: Segmentation of ultrasound B-mode images with intensity inhomogeneity correction. IEEE Trans. Med. Imag. 21(1), $48-57(2002)$ 